Nat Rev Clin Oncol. 2013 August ; 10(8): 460-471. doi:10.1038/nrclinonc.2013.100.

\title{
Minimal Residual Disease in Acute Myeloid Leukemia
}

\author{
Christopher S. Hourigan ${ }^{1}$ and Judith E. Karp ${ }^{2}$ \\ ${ }^{1}$ Myeloid Malignancies Section, Hematology Branch, National Heart, Lung and Blood Institute, \\ Bethesda, MD \\ ${ }^{2}$ Sidney Kimmel Comprehensive Cancer Center at Johns Hopkins, Baltimore, MD
}

\begin{abstract}
Technological advances in the laboratory have lead to substantial improvements in clinical decision-making by the use of pre-treatment prognostic risk stratification factors in acute myeloid leukemia (AML). Unfortunately similar progress has not been made in treatment response criteria, with the definition of "complete remission" in AML largely unchanged for over half a century. Several recent clinical trials have demonstrated that higher sensitivity measurements of residual disease burden during or after treatment can be performed, that results are predictive for clinical outcome and can be used to improve outcomes by guiding additional therapeutic intervention to patients in clinical complete remission but at increased relapse risk. We review here these recent trials, the characteristics and challenges of the modalities currently used to detect minimal residual disease (MRD), and outline opportunities to both refine detection and better clinically utilize MRD measurements. MRD measurement is already the standard of care in other myeloid malignancies such as chronic myelogenous leukemia (CML) and acute promyelocytic leukemia (APL). It is our belief that response criteria for non-APL AML should be updated to include assessment for molecular complete remission ( $\mathrm{mCR}$ ) and that recommendations for postconsolidation surveillance should include regular monitoring for molecular relapse as a standard of care.
\end{abstract}

\section{1) Introduction}

Our understanding of heterogeneity and the underlying disease biology of the acute myeloid leukemia (AML) has been greatly deepened by the recent use of modern laboratory advances ${ }^{1-4}$ which have in turn allowed more refined pre-treatment risk stratification using molecular prognostic ${ }^{5,6}$ and predictive ${ }^{5}$ biomarkers. Unfortunately this molecular revolution has been incompletely translated. Just as the most commonly used induction chemotherapy treatment for adult non-APL AML has not changed substantially in the past forty years 7,8 the treatment response criteria for a "complete remission" (CR) has not dramatically changed in almost sixty years ${ }^{9,10}$ (Table 1 ).

It has been recognized since at least 1969 that a clinical "complete remission" is an early, important, necessary but insufficient step on the path to long-term disease control in acute

Correspondence: Christopher S. Hourigan, Myeloid Malignancies Section, National Heart, Lung and Blood Institute, Room 6C-103C, 10 Center Drive, Bethesda, Maryland 20892-1583; hourigan@nih.gov.

The authors report no conflicts of interest. 
myeloid leukemia (AML) ${ }^{11}$. While a patient at first presentation with high blast count AML may have up to $10^{12}$ leukemic cells (equivalent to several pounds of tumor), that same patient after induction chemotherapy treatment judged to be in an initial "complete remission" may have anywhere as few as none and as many as $10^{10}$ leukemic cells remaining (a number of cells equivalent to a two cubic centimeter tumor mass in a solid tumor setting). It is clear that due to the low sensitivity criteria used that some "complete remissions" are more complete than others (Figure 1). Those in this highly diverse state of "complete remission" currently have clinical decisions regarding subsequent treatment (to establish if the risks associated with stem cell transplantation (SCT) as consolidation therapy are outweighed by a potential benefit from reduction in relapse risk ${ }^{12}$ ) made by stratification solely on the basis of pre-treatment risk factors ${ }^{5,6}$ rather than any direct quantification of remaining leukemic disease burden.

Many patients diagnosed with AML are not eligible, due to age or comorbidity, for intensive chemotherapy ${ }^{13}$. In those who can tolerate such treatment however initial CR rates of greater than $65 \%$ in newly diagnosed poor risk leukemias ${ }^{14,15}$ and up to $92-99 \% 16,17$ in those with core binding factor (CBF) AML are now achievable with optimal induction therapy which has shifted the focus to the prediction and prevention of relapse.

The current "standard of care" pre-treatment risk factor stratification assigns patients in an initial complete remission to a risk of relapse based on the average clinical outcomes of large historical populations who had similar pre-treatment cytogenetic and molecular characteristics in their leukemia at presentation ${ }^{6,10}$ and does not include any patientpersonalized assessment of induction treatment efficacy by high-sensitive detection of residual leukemia burden at the time that decisions regarding most appropriate consolidative treatment must be made.

This ability to measure minimal residual disease (MRD) in AML was not available in 1956 when criteria for the evaluation of response to treatment in acute leukemia were proposed 9 and so understandably thresholds were set based on the technology available at that time (Table 1). MRD has however been measurable in AML patients in a clinical CR via quantitative reverse transcriptase polymerase chain reaction (RQ-PCR) ${ }^{18}$ or flow cytometry (FC) ${ }^{19}$ for over twenty years now. Technologies used to detect MRD in acute leukemia have included traditional expert microscopy for morphology, fluorescence in situ hybridization (FISH), cytogenetics, flow cytometry (FC), gene expression analysis using RQ-PCR (GEPCR), PCR for translocations, rearranged or mutant sequence (Mut-PCR), DNA sequencing and in the setting of monitoring post stem cell transplantation (SCT) analysis of chimerism by PCR or FISH (Figure 1, Table 2, reviewed in ${ }^{20-24}$ ). There is already excellent evidence for clinical decision making as a standard of care based on multi-parameter flow cytometry (FC) in acute lymphoblastic leukemia (ALL) ${ }^{25}$ and reverse transcriptase-polymerase chain reaction analysis (RT-PCR) in Acute Promyelocytic Leukemia (APL) ${ }^{26-28}$ so the remainder of this review will therefore focus solely on the use and opportunities for use of MRD in non-APL AML.

In this article we compare the modalities of MRD detection currently in use in AML by reviewing twelve recently reported clinical trials or series (Table 3) for what they teach us 
regarding the optimal technology, indication, timing and predictive and prognostic utility of such MRD measurements. We conclude by discussing clinical scenarios where we believe MRD measurement in AML could have immediate utility.

\section{2) MRD Detection by Gene Expression Analysis}

The development of quantitative real time polymerase chain reaction (RQ-PCR) technology has provided many logistical and technological advantages compared to other MRD detection methodologies (Table $2,{ }^{20}, 29,30$ ). The adaptation of this highly sensitive and specific technology to the quantification of disease burden in chronic myeloid leukemia ${ }^{31,32}$ was responsible for fundamental contributions to drug development ${ }^{33,34}$, response criteria and treatment guidelines ${ }^{35,36}$ and detection and analysis of treatment failures ${ }^{37,38}$ in that disease. Unfortunately no single rearranged or mutated target sequence analogous to the $b c r$ $a b l$ fusion transcript is found in all cases of AML, motivating the search for genes expressed at higher levels in AML than normal tissues that could serve as a target for RQ-PCR. While a variety of such genes (eg: leukemia associated antigens ${ }^{39}$ ) are known and could be potentially used for this purpose, much of the work has focused on the use of WT1 ${ }^{40-52}$.

WT1 is zinc finger transcription factor with an essential role in normal development with expression in the adult restricted to the renal glomerular podocytes and a subset of hematopoietic precursors ${ }^{53}$. It is overexpressed at the mRNA level in 80-90\% of AML cases at diagnosis in both peripheral blood (PB) and bone marrow aspirate (BM), which has lead to its adoption as a standardized method for MRD detection using RQ-PCR ${ }^{48}$. This European LeukemiaNet study found the magnitude of WT1 log reduction after induction chemotherapy to be an independent predictor of relapse $(<2 \log$ reduction was associated with a $75 \%$ risk of relapse at 5 years compared to only $40 \%$ risk in those with a $\geq 2 \log$ reduction), unfortunately only $46 \%$ of 238 patient peripheral blood pretreatment samples examined had WT1 sufficiently overexpressed to allow the detection of a reduction of that magnitude. PB appeared preferable to BM for subsequent monitoring as higher levels of WT1 mRNA expression in healthy donor BM (median $19.8 \mathrm{WT} 1$ copies $/ 10^{4} \mathrm{ABL}$, range: 0213) compared to healthy donor PB (median $0.01 \mathrm{WT} 1$ copies/104 ABL, range: 0.01-47.6) limited the working dynamic range for detection. Patients with WT1 levels above the upper limit of normal (250 copies $/ 10^{4} \mathrm{ABL}$ in $\mathrm{BM}$ or 50 copies $/ 10^{4} \mathrm{ABL}$ in $\mathrm{PB}$ ) after consolidation therapy were found at a significantly increased risk of relapse $(67 \%$ vs. $42 \%$ at 5 years $)^{48}$.

Within the past year there have been some direct "head-to-head" comparisons of WT1 RQPCR assessment with other MRD modalities. Kwon et. al., described 21 AML patients followed after allogeneic SCT using MRD monitoring with WT1 RQ-PCR gene expression analysis, chimerism and flow cytometry ${ }^{52}$. RQ-PCR was analyzed using ddCT method using GUS reference gene and K562 calibrator sample wsith $>0.025 \%$ and $>0.55 \%$ considered positive results after two consecutive measures in $\mathrm{PB}$ and BM respectively. Four-color flow cytometry was performed, using least $10^{6} \mathrm{BM}$ cells, with a leukemic aberrant phenotype of $>0.1 \%$ considered positive. All of nine relapses (range 50-560 days after SCT) were detectable using WT1 monitoring, in eight cases this "molecular relapse" 23 preceded clinical (ie: hematological) relapse by a median of 137 days (range: 52-462). Three of these patients 
had continuously elevated WT1 levels (ie: molecularly refractory) while six were initially negative after SCT followed by a molecular relapse and hematological relapse. Eight of these nine patients had molecular relapse detectable from peripheral blood sampling, including all three patients with extra-medullary relapse. In no case was relapse detected by flow cytometry or mixed chimerism prior to WT1 positivity (although it should be noted that fewer timepoints were examined using these methodologies). A tenth patient also had elevated WT1 levels after SCT but had not relapsed by the time of publication. All eleven patients without WT1 overexpression after SCT remained relapse free.

Miyazaki et. al. recently compared the use of FC with quantitative PCR for WT1 and/or leukemia-specific fusion transcripts in 41 acute leukemia patients after allo-SCT ${ }^{54}$. In their analysis of 156 samples they demonstrated good concordance (71.8\%) between FC and WT1 PCR based MRD detection, with most of the discordance from samples that were PCR positive for WT1 but negative by FC. Unfortunately this report included a mixture of patients with AML $(n=31)$ and ALL $(n=10)$ making additional interpretation challenging.

Rossi et. al. reported in 2012 the use of six color flow cytometry with $>0.1 \%$ leukemiaassociated immunophenotypes (LAIPs) in BM considered positive MRD compared to WT-1 gene expression profiling using RQ-PCR ${ }^{51}$. In this single hospital series, of 47 consecutive AML patients achieving a CR after induction chemotherapy, 23 were eligible for this study as they did not have leukemia-specific rearranged or mutated genetic sequence suitable for MRD assessment. This study concluded that FC and WT-1 RQ-PCR "showed comparable capacity in terms of technical performance and clinical significance to identify high risk patients who eventually relapsed", a surprising finding given that testing was not restricted to those $~ 50 \%$ of patients with WT- 1 mRNA elevated to a level where a two log variation could be detected (the mean expression of 3320 copies WT1/10 4 ABL in diagnosis samples would allow on average only slightly more than 1 log dynamic range dramatically limiting the sensitivity), RQ-PCR testing was performed on bone marrow, and the threshold for positive MRD ( 90 copies $/ 10^{4} \mathrm{ABL}$ ) was set almost two thirds lower than reported in the prior standardization of this assay ${ }^{48}$. Given these factors it is possible that this study compared FC with a conservative estimate of the optimal performance characteristics of the WT-1 assay.

\section{3) MRD in core binding factor AML}

The core binding factor (CBF) AMLs, defined as those carrying either the $t(8,21)$ chromosomal translocation (RUNX1-RUNX1T1 fusion gene) or $\operatorname{Inv}(16) / t(16 ; 16)$ rearrangement (CBFB-MYH11 fusion gene), like APL AML ${ }^{26-28}$, represent ideal AML subtypes for RQ-PCR based MRD monitoring due to presence of a defined uniquely leukemia-specific stable molecular target. The CBF AMLs represent up to approximately $20 \%$ of all AMLs in adults and are considered to be "better-risk" or "favorable risk" status based on cytogenetics and molecular stratification $5,6,10$. Additional prognostic information in these subsets would be easily actionable; chemotherapy is often preferred for consolidation so treatment escalation to stem cell transplantation would be an option for those at the highest risk of relapse ${ }^{10,12}$. Additionally, compared to other AML subtypes 
effective salvage therapy is available for Inv(16) AML making post-treatment monitoring for early detection of relapse particularly important ${ }^{55}$.

The AML02 trial $^{56}$ was a multicenter trial that treated 216 children for AML using risk directed therapy based on presentation leukemia genetic factors and FC based MRD which achieved impressively high rates of CR (94\% after induction 2), 3 year event free survival (EFS) (63\%) and 3 year overall survival (OS) (71\%). FC-based MRD levels after induction therapy were important predictors of relapse, with FC MRD >1\% after induction 1 identified in multivariate analysis as being an independent adverse prognostic factor for both EFS and OS. A subsequent report in $2012^{57}$ retrospectively analyzed 508 samples from 77 of these patients who had leukemia associated rearranged sequence (RUNX1-RUNX1T1, CBFBMYH11 or MLL fusion transcripts) suitable for detection by PCR. There was good correlation between negative PCR results and FC, of 311 samples classified negative by PCR 308 were negative by FC. Discrepancy was observed between positive PCR results and FC, only 27 of 197 positive PCR results were confirmed by FC, this however may be an artifact of the technology used to process earlier samples, when modern RQ-PCR technology was used there was excellent correlation between PCR and FC results in 138 of 139 samples tested.

The British MRC15 trial also reported in 2012 the results of prospective assessment of MRD using RQ-PCR for RUNX1-RUNX1T1 or CBFB-MYH11 transcripts in 278 patients aged 15-70 treated for CBF AML ${ }^{16}$. MRD was informative not only in detection of molecular relapse preceding hematological relapse in serial monitoring during remission, but also the result of a single MRD assessment after completion of induction therapy while in complete remission was itself prognostic for subsequent relapse risk in a multivariate analysis that included age, WBC, secondary AML, performance status and sex. After completion of induction chemotherapy to CR those $46 \%$ of patients with $t(8,21)$ who had achieved a $3 \log$ reduction in BM MRD had a cumulative incidence of relapse (CIR) of only 4\% (1 relapse in 28 patients) compared to a CIR of $32 \%$ in the patients who did not achieve this level of initial leukemic tumor burden reduction. Absolute levels of MRD were also significant with a threshold of $<100$ copies in BM (47\% of patients) identifying a cohort with a CIR of just $7 \%$ and the threshold of 1000 copies in PB was able to discriminate patients after induction into low (78\% of patients, CIR 15\%) and high (22\% of patients, CIR 50\%) risk groups. MRD assessment while in CR after induction therapy for inv(16) AML also allowed patients to be stratified based on PB copy number into low (<10 copies, $51 \%$ of patients, CIR $21 \%$ ), intermediate (10-500 copies, CIR of 56\%) and high (CIR of $100 \%$ for those with $>500$ copies) risk groups for subsequent relapse. Subsequent serial monitoring during remission after completion of all therapy for $t(8,21)$ detection of $>100$ copies of RUNX1-RUNX1T1 transcript in PB or $>500$ copies in BM (ie: molecular relapse) was associated with a hematological relapse rate of $100 \%$ compared to only $7 \%$ in those below this threshold. Similar thresholds could be established as significantly prognostic of relapse risk for Inv(16) patients in CR after completion of therapy by MRD monitoring in BM ( $>50$ copies, $32 \%$ of patients, $100 \%$ hematological relapse rate compared to a CIR of $10 \%$ in those with $<50$ copies) and PB (>10 copies, $28 \%$ patients, $97 \%$ hematological relapse risk compared to a CIR of $7 \%$ in those with $<10$ ). 
Finally, the French CBF-2006 trial reported in early 2013 the results of treatment of 198 adults aged 18-60 with newly diagnosed CBF AML using prospective evaluation of pretreatment leukemia molecular factors (FLT3, KIT and RAS mutations) with MRD assessment for relapse risk-stratification ${ }^{17}$. MRD levels were determined from BM using RQ-PCR prior to three monthly consolidation cycles of cytarabine of $3000 \mathrm{mg} / \mathrm{m} 2 / 12 \mathrm{hr}$ on day 1, 3 and 5 followed by GCSF from day 8 to neutrophil recovery. Patients were randomized to one of two induction regimens resulting the initial post-induction conventional (hematological) CR rates of $98-100 \%$. MRD assessment however could distinguish between the efficacy of these induction regimens, demonstrating differences in the depth of remission and the residual disease burden after induction $(0.10 \%$ vs. $0.26 \%$ fusion transcript ratios) in patients in CR, a difference which appeared to persist after subsequent consolidation cycles. Patients in CR who had not achieved a $3 \log$ reduction in MRD after the first cycle of consolidation therapy became eligible on this protocol for stem cell transplantation, that level of reduction was not reached in $24 \%$ and $35 \%$ of patients on the two arms. Univariate analysis of prognostic factors of relapse risk identified higher white blood cell count (WBC), KIT mutations, FLT3 mutations, less than a $3 \log$ reduction in MRD after first cycle of consolidation (MRD2) and an absolute MRD2 level of $>0.1 \%$ as associated factors. Multivariate analysis of WBC, KIT and FLT3 mutations, and MRD2 response showed that MRD2 response (by $3 \log$ reduction or absolute level) was the sole factor influencing specific hazard of relapse both in the whole patient cohort, and also in the individual $t(8,21)$ and $\operatorname{Inv}(16)$ subsets. This suggestion that when prognosticating relapse risk the reality of disease burden after treatment (MRD) may trump molecular markers of disease biology assessed prior treatment is provocative, and counter to current clinical practice $^{6,10}$.

\section{4) PCR for mutated or rearranged sequence for MRD in non-CBF AML}

As seen with $\mathrm{CML}^{31,36,38}$, $\mathrm{APL}^{26-28}$ and $\mathrm{CBF} \mathrm{AML}^{16,17,56}$, rearranged or mutated genetic sequence targets allow for high sensitivity RQ-PCR based detection of leukemic burden. While no universal target exists for AML several gene mutations are common ${ }^{5,6}$. Nucleophosmin (NPM1) gene mutations are seen in 25-30\% of cases of AML ${ }^{58}$ and appears to be a stable marker for MRD monitoring 59,60 . The German-Austrian AML study group recently reported on the prospective use of NPM1 ${ }^{\text {mut }}$ MRD in 245 adult (age 19-61) AML patients ${ }^{58}$. The degree of disease burden as measured by NPM1 ${ }^{\text {mut }}$ RQ-PCR MRD was significantly associated with prognosis for patients in a complete remission after each cycle of treatment. For example, MRD negativity after completion of induction therapy was associated with a 4 year CIR of only $6.5 \%$ (achieved by 26 patients) compared to a CIR rate of 53\% in the 111 patients in morphological CR but not achieving this molecular CR (mCR) milestone. Additionally, 4 year CIR was $15.7 \%$ (OS: 80\%) in the $62 \mathrm{CR}$ patients who were $\mathrm{MRD}^{\text {neg }}$ are the completion of therapy compared to a CIR of $66.5 \%$ (OS: 44\%) in those in CR but MRD ${ }^{\text {pos }}$. While BM MRD assessment was preferable during therapy, PB MRD showed good concordance (88\%) with BM in post treatment monitoring, when exceeding a threshold of 200 copies NPM1 ${ }^{\text {mut }} / 10^{4} \mathrm{ABL}$ was $100 \%$ predictive of relapse. Of note, only $9 \%$ of evaluable relapses had no or minimally detectable NPM1 ${ }^{\text {mut }}$ at the time of relapse, confirming the relative stability of this target. Similar results were shown by Miglino et. al., 
where achievement of a NPM ${ }^{\text {mut }}$ molecular CR after induction was associated with a statistically significant lower relapse risk than those who remained with detectable disease, and a statistically significant higher overall survival at 36 months $(64.3 \% \text { vs. } 11.9 \%)^{61}$. NPM1 ${ }^{\text {mut }}$ molecular relapse was invariably associated with eventual hematological relapse $^{61}$. Very recent data from a large 174 patient dataset has determined that specific NPM1 ${ }^{\text {mut }}$ MRD threshold levels can be determined in CR1, during surveillance after chemotherapy and after allo-SCT that are significantly associated with poor overall and disease free survival. Importantly, this work also showed that sampling from peripheral blood can take the place of bone marrow testing for this MRD assay ${ }^{62}$.

Despite evidence that the approximately $50 \%$ of AMLs with sufficient WT1 overexpression for MRD monitoring includes many cases of NPM1 ${ }^{\text {mut }}$, Inv(16) or adverse cytogenetic $\mathrm{AML}^{48}$, it is nevertheless likely that a validated and stable molecular target for RQ-PCR MRD monitoring can be found for the majority (perhaps 65-75\%) of patients diagnosed with AML. Additional stable molecular markers with potential for development for MRD monitoring include DNMT3A ${ }^{63}$ and CEBPA ${ }^{64}$. Despite initial concerns that tracking FLT3ITD mutation would not be informative due to concerns regarding stability ${ }^{65}$ using more sophisticated techniques ${ }^{66}$ this mutation has been shown to be highly predictive for relapse risk $^{67,68}$. It is likely therefore that clinical laboratories will have to offer, or contract with a reference laboratory for, a portfolio of RQ-PCR MRD assays, which will increase as next generation sequencing (NGS) technology increases the number of viable molecular targets ${ }^{3}$. Once whole genome and/or exome sequencing of tumor and germline becomes possible routinely at cancer diagnosis ${ }^{69}$ this will facilitate identification of not only patient-specific MRD markers ${ }^{70}$, but also allow molecular diagnosis ${ }^{71}$, subsequent retrospective discovery of prognostic factors ${ }^{5}$, and triage to the most appropriate trials of targeted agents ${ }^{72}$. Finally, the ability to identify stable molecular targets within the leukemic stem cell population ${ }^{73-75}$ for MRD monitoring and therapeutic targeting is of obvious great interest.

\section{5) Flow Cytometry for MRD in AML}

Flow cytometry is, when used by appropriate experts, an excellent method for determining MRD in AML with high sensitivity and specificity 22, 56, 76-86. Some of the advantages and logistical challenges associated with this method are outlined in Table 2. Modern flow cytometry based MRD detection is most commonly based on the definition aberrant cell surface marker expression in AML cells as a leukemia associated phenotype (LAP or leukemia associated immunophenotype, LAIP) that is not, or only infrequently, seen in normal or recovering bone marrow or blood ${ }^{79,87}$. These can include asynchronous expression of early and late antigens, cross lineage infidelity (eg: expression of lymphoid markers on myeloid cells) and antigen under or over expression ${ }^{79,81}$. Such LAIPs can be personalized to each patient at presentation, with the caveat that AML is a clonal disease and relapsed disease may have a different immunophenotype ${ }^{80,88-91}$.

The main advantage of flow cytometry in MRD monitoring is its near universal applicability; one recent study ${ }^{92}$ found that $94 \%$ of patients had a suitable LAIP for use, with the range reported in the literature when at least three color FC has been used ranging between $60-100 \%{ }^{79}$. We avoid in this review the ungainly terminology of "immunological 
relapse" and "immunological complete remission" when describing the reappearance or absence of MRD as detected by flow cytometry, the levels of MRD necessary for the molecular response criteria described by Hokland and Ommen ${ }^{23}$ could however, in principle, also be detected by modern flow cytometry. It is unclear however if quantitative MRD results can be compared across multiple centers given the importance of human and technical factors ${ }^{24}$ in interpretation, indeed even in the context of a recent clinical trial with identical standard operating procedures, reagents and instruments used variation was seen between four reference laboratories ${ }^{81}$.

Given their extremely successful experience of using FC for ALL MRD 25 those treating pediatric patients have been leaders in using this technology in AML, including the AML02 study already discussed ${ }^{56}$ where subsequent analysis determined that flow cytometry based MRD measurement after first and second induction was statistically associated with five year event-free and overall survival ${ }^{57}$.

A cohort of 219 AML patients under the age of 21 treated on the Childrens Oncology Group (COG) trial AAML03P1 was recently analyzed ${ }^{93}$ for MRD using four color FC using a nonLAIP based "difference from normal" approach" ${ }^{94}$. In 188 patients with initial morphological CR after the first induction phase the presence of FC defined MRD (seen in $25 \%$ of patients) was highly correlated with relapse (3yr relapse risk $60 \%$ vs. $29 \%$ in MRD $^{\text {neg }}$ patients). Cytogenetic and molecular risk factors were associated with risk of MRD while in CR after first induction with $11 \%, 29 \%$ and $50 \%$ of those with favorable, intermediate and high-risk cytogenetics being positive for MRD at this timepoint and 25\% of those with FLT3-ITD being MRD ${ }^{\text {pos }}$ compared to $0 \%$ of those with NPM1 mutations. A multivariate analysis that included molecular risk group stratification and presentation WBC as variables found that MRD positivity after first induction was an independent predictor of relapse free survival (RFS) for those patients in morphological CR. Interestingly of the 27 patients who did not achieve a morphological CR (>5\% blasts) after first induction therapy, seven were found to be negative for residual disease by FC and clinically remain long-term survivors. Finally, highlighting the increased logistical and resource implications of providing high-quality FC based MRD assessment, this study required all analyses to be performed by 2 independent analysts with concordance necessary for MRD diagnosis.

Flow cytometry for MRD in adult AML patients can also be prognostic. A retrospective analysis of 123 adults enrolled in EORTC/GIMEMA protocols in the period 1998-2008 in a single center, showed that FC-based MRD at the end of consolidation could blur distinctions between pre-treatment cytogenetic and molecular risk groups ${ }^{95}$. For example, after consolidation, patients in CR from good risk karyotype AML but MRD ${ }^{\text {pos }}$ had a 4 year RFS of only $15 \%$ compared to those in $\mathrm{CR}$ and $\mathrm{MRD}^{\text {neg }}$ from intermediate risk karyotype AML who had a 4 yr RFS of $63 \%$. The use of post-consolidation FC-based MRD status combined with pre-treatment cytogenetics and FLT3 mutational status could segregate patients into groups with low and high risk for subsequent relapse. Further analysis focused on the effect of autologous (auto-SCT) or allogeneic (allo-SCT) stem cell transplantation in the 79 patients who received one of these therapies as post-consolidation therapy. There was no statistical difference in outcome in low risk patients (but 5 non-relapse, transplant related, deaths in the allo-SCT group) but superior outcomes after allo-SCT for patients in the high 
risk category (RFS $47 \%$ vs. 13\% auto-SCT) suggesting MRD-directed therapy escalation can improve outcomes.

Within any individual patient AML is not a single homogenous disease at the level of genetics and biology $1,3,4$. Importantly Gerber et. al., recently identified using FC a population of cells that while rare in leukemia at diagnosis, appeared significantly overrepresented in the MRD fraction in patients in a morphological CR after treatment ${ }^{74}$. These cells were capable of producing complete leukemic engraftment in an immunodeficient mouse model of stem cell function. The presence of such cells in patients after treatment was highly correlated with subsequent clinical relapse. This important finding is now the basis of an ongoing clinical trial at Johns Hopkins Hospital to determine the 2year RFS of AML patients with these "leukemic stem cells" (LSC) at first CR who receive either chemotherapy or stem cell transplantation (NCT01588951). The tracking of LSC MRD represents another unique advantage of flow cytometry until such a time when unique molecular markers of such cancer stem cells can be identified; this is an area of much interest $^{3,73-75,96-103}$.

\section{6) Use of MRD in the setting of Allogeneic Transplantation}

Allogeneic stem cell transplantation remains the first and most effective immunotherapy used for hematological malignancies ${ }^{39}$ and the possibility of using MRD to enable both appropriate triage of patients pre-transplantation based on their individual risk of relapse and to follow patients and treat prior to frank clinical/hematological relapse is of obvious interest ${ }^{95,104-108}$. In addition to those studies already discussed there are several other reports that directly address the question of the role of MRD testing in the peri-transplant setting. Walter et. al., showed, using a "difference from normal" FC based MRD approach, in patients in conventional CR prior to allo-SCT that $2 \mathrm{yr}$ relapse rate $(64.9 \%$ vs. $17.6 \%)$ and overall survival (30.2\% vs. 76.6) were markedly different in the group positive and negative for MRD respectively. This increased mortality risk in patients MRD ${ }^{\text {pos }}$ before allo-SCT remained statistically significant even when adjusting for other factors ${ }^{78}$.

This worse outcome in patients who are in conventional CR but are MRD ${ }^{\text {pos }}$ prior to alloSCT has also been seen when using cytogenetic ${ }^{109}$ and WT-1 PCR MRD testing $50,105,110$. For example, Pozzi et. al., just reported on the use of WT1 PCR to predict relapse risk in 122 AML patients the allo-SCT setting ${ }^{105}$. They found, using a threshold of 100 WT1 copies $/ 10^{4}$ copies of ABL, that MRD testing using WT1 levels pre-transplant (69 MRD ${ }^{\text {neg }}$ patients had relapse rate of $26 \%$ vs. relapse rate $53 \%$ in 53 patients who were MRD ${ }^{\text {pos }}$ ), and especially post-transplant (only $16 \%$ of $55 \mathrm{MRD}^{\text {neg }}$ patients relapsed, compared to $55 \%$ of $67 \mathrm{MRD}^{\text {pos }}$ patients) was highly predictive of relapse risk. Combining information from preand post- transplant timepoints appeared to be especially informative.

\section{7) Can MRD guide therapy to improve outcomes?}

Beyond providing patients with prognostic information, detection of residual disease is only truly useful if therapeutic intervention is available that can change the course of the disease. Evidence for effective MRD-based risk-directed therapy has already been discussed; in AML02 $2^{56}$ levels of MRD during induction were used to determine timing and need for 
escalation of induction therapy and then a combination of initial risk classification and MRD based assessment of induction response was used to assign appropriate consolidation therapy. This MRD-directed approach resulted in superior outcomes compared to prior trials with 3 year EFS (63\%) and OS (71\%).

Multiple reports of treatment of molecular relapse prior to clinical/hematological relapse have now shown that MRD-directed therapy can lead to improved clinical outcomes. CD34+ donor chimerism analysis is a specific method for monitoring for MRD after allo-SCT ${ }^{111}$ with a chimerism level falling below $80 \%$ after SCT having been shown to be a harbinger of hematological relapse in a median of 61 days ${ }^{112}$ The RELAZA trial ${ }^{113}$ demonstrated that 4 cycles of azacitidine therapy for MDS/AML patients with CD34+ chimerism falling below this level could delay hematological relapse to a median of 231 days and lead to continuous RFS in $20 \%$ of patients without any further treatment. Analogously, it has been shown that rising NPM1 ${ }^{\text {mut }}$ MRD levels are predictive of upcoming hematological relapse in a median time of eight weeks ${ }^{60}$. Sockel et. al., treated ten AML patients in CR but with increasing NPM1 ${ }^{\text {mut }}$ MRD levels with azacitidine therapy with a resulting molecular response (at least $1 \log$ reduction in MRD levels) in 7 of these patients and 7 patients remaining in a complete hematological response after a median follow-up of ten months ${ }^{114}$.

In the Pozzi study already discussed ${ }^{105}$ retrospective analysis suggested that patients with rising WT1 MRD who received immunotherapy intervention in the form of donor lymphocyte infusions (DLI) had a better 5 year overall survival (44\%) than those with rising WT1 MRD levels who did not receive DLI. This non-randomized study however has several confounding factors however that make interpretation challenging, exacerbated by the fact that time to hematological relapse was not different between the two groups. There is strong evidence however that DLI can be an efficacious intervention in the setting of MRD relapse. Yan et. al. ${ }^{115}$ analyzed data from 814 patients aged 2-59 receiving SCT (for AML, ALL or MDS-RAEB) from a single center in the period 2006-2010. The 105 patients who were MRD $^{\text {pos }}$ by FC or WT1 RQ-PCR after SCT were treated with either IL-2 and/or modified DLI. Presence of MRD after SCT was associated with greater 3yr CIR (46\% vs. 18.1\%). Significant differences at 3years were seen within the MRD ${ }^{\text {pos }}$ group based on the modality used to treat their molecular relapse; CIR of $64.4 \%$ (OS of 28.1\%) in those treated with IL-2 alone compared to CIR of only $27.8 \%$ (OS of $58.3 \%$ ) in those treated with DLI. In fact, CIR and disease free survival was not statistically different between the MRD ${ }^{\text {neg }}$ patients and those MRD ${ }^{\text {pos }}$ patients treated with DLI. Unfortunately responses for MDS/AML patients were not reported separately from those in ALL patients.

The same group has just reported their experience of MRD-directed therapy for patients with $\mathrm{t}(8,21) \mathrm{AML}^{116}$. MRD determined by RUNX1/RUNX1T1 transcript levels by RQ-PCR was shown to be predictive for relapse when measured after the second cycle of consolidation. A non-randomized comparison of those in a high relapse risk group (defined by being in a morphological CR but MRD ${ }^{\text {pos }}$ after $2^{\text {nd }}$ consolidation) who received allo-SCT as mandated by risk-directed protocol $(n=40)$ compared with those who did not due to patient preference or lack of appropriate donor $(\mathrm{n}=29)$ showed a substantial benefit to this escalation of therapy (CIR $22.1 \%$ vs. $78.9 \%, \mathrm{p}<.0001$ ). This benefit to allo-SCT was not seen however in the low risk (CR, MRD ${ }^{\text {neg }}$ after $2^{\text {nd }}$ consolidation) group. These observations need confirmation in a 
randomized trial but add to the increasing body of evidence that not only is MRD prognostic, but that therapy based on MRD may improve clinical outcomes.

\section{8) Conclusion - how MRD can help now}

Despite the challenges in standardizing definitions of assay and AML specific thresholds of MRD and the lack of one universal modality or target, there is now ample indisputable evidence that the MRD monitoring can improve relapse risk stratification and detection in AML. The use of MRD to guide treatment is already an accepted standard of care in acute lymphoblastic leukemia ${ }^{25}, \mathrm{CML}^{35}$ and $\mathrm{APL}^{26,28}$ and based on recent studies discussed here $^{16,17}$ will also soon be in CBF AML ${ }^{117}$.

In the recent CBF AML MRD trials the average kinetics of relapse appeared to be approximately $0.5-0.6 \log$ per month, MRD was checked 3-4 monthly in peripheral blood in the first two year period when most relapses occurred and the median lead time between molecular relapse and hematological relapse was 3 to 4.5 months ${ }^{16}$. While disease biology may influence the kinetics of relapse ${ }^{23}, 49,67,118$ and the sensitivity of the MRD detection modality used will influence the lead-time between molecular and hematological relapse ${ }^{24}$ it now seems clear that regular MRD monitoring could be easily integrated into the current recommendations for post-consolidation surveillance of a peripheral blood draw for complete blood count with platelets every $1-3$ months for the first two years ${ }^{10}$ and that doing so would provide a therapeutic window for intervention before frank clinical relapse.

The search for optimal MRD assessment in other AML types will be an iterative process and the understandable quest for the perfect should not become the enemy of the introduction of the better; even low sensitivity MRD to a modest $10^{-3}$ or $10^{-4}$ detection threshold (see figure 1) may provide more actionable information than traditional hematological $\mathrm{CR}^{119}$. There are a number of specific clinical scenarios were the adoption of routine standard of care MRD monitoring could make an immediate impact (Table 4).

MRD thresholds need not be infallibly associated with evitable hematological relapse to be clinically useful, the concept of maintenance therapy for prevention of relapse in those at increased risk is already under investigation in myeloma ${ }^{120}$, follicular lymphoma ${ }^{121}$ and lung cancer ${ }^{122}$ and as less toxic therapies with efficacy in leukemia become available ${ }^{39,123-126}$ identification of patients at the greatest risk of relapse who may benefit from such additional therapy in the post-conventional treatment setting would be particularly useful. Similarly, monitoring for the early detection of the development of subclinical disease would also have utility for those therapies including immunotherapies where optimal effects might be observed in lower disease burden states $39,73,127,128$.

Standardized mCR criteria will likely also aid drug development. Hematological CR is a blunt tool to evaluate differences between therapies and MRD measurement offers greater dynamic range to detect changes in leukemia burden ${ }^{17}$ and monitor for treatment effect on leukemic subpopulations that may be associated with relapse $\mathrm{e}^{4,74}$. The ability to dose escalate or de-escalate therapy based on MRD response may increase the number of AML patients able to therapy with curative intent, currently many of those diagnosed do not 
receive best available therapy ${ }^{13}$ and may benefit from the titration of safe, moderately effective therapy based on the objective high sensitivity response criteria of MRD.

How the results of MRD assessments are ultimately integrated into standard cytogenetic and molecular risk stratification systems remains an open question ${ }^{129}$ that ultimately will only be answered be prospective clinical trials; while there is clear evidence that disease biology in the form of pre-treatment risk stratification factors influence risk of having residual disease after initial treatment ${ }^{93}$ it appears that deep negativity in MRD measurements post-treatment is an independent protective factor against relapse and that this factor may trump other considerations in some ${ }^{17}$ but not all ${ }^{95}$ situations.

Many recent excellent reviews, editorials and comment pieces have advocated for the incorporation of MRD monitoring into AML clinical trials $20,22-24,30,130-134$. It is our hope that by reviewing the most recent clinical trial results in this area we may add our voices to this chorus. While much work remains to standardize the timing, performance, indications and application of MRD monitoring across multiple centers this should not preclude, in the meantime, the more immediate establishment of local AML MRD monitoring capacity. Given our belief that the current standard of care therapy for patients in the USA diagnosed with acute myeloid leukemia, if treated with curative intent, is referral to a specialized center where an appropriate clinical trial can be offered ${ }^{135}$, we would add now an additional characteristic of such a leukemia center of excellence would be the capacity to routinely provide MRD monitoring for the majority of cases of AML and to universally integrate such monitoring into all future therapeutic clinical trials.

\section{Acknowledgments}

The authors wish to thank Drs Gouri Bardhan, A. John Barrett, Minoo Battiwalla, Luis Diaz and and Sandhya Panch for useful discussions and Alan Hoofring of the NIH Medical Arts Service for assistance in creating Figure 1.

This research was supported by National Institutes of Health grant P30CA006973 and the Intramural Research Program of the NIH, National Heart, Lung and Blood Institute.

Any views expressed here represent personal opinion and do not necessarily reflect those of the U.S. Department of Health and Human Services, or the United States federal government.

\section{References}

1. Walter MJ, et al. Clonal architecture of secondary acute myeloid leukemia. N Engl J Med. 2012; 366:1090-8. [PubMed: 22417201]

2. Parkin B, et al. Clonal evolution and devolution following chemotherapy in adult acute myelogenous leukemia. Blood. 2012

3. Welch JS, et al. The origin and evolution of mutations in acute myeloid leukemia. Cell. 2012; 150:264-78. [PubMed: 22817890]

4. Ding L, et al. Clonal evolution in relapsed acute myeloid leukaemia revealed by whole-genome sequencing. Nature. 2012; 481:506-10. [PubMed: 22237025]

5. Patel JP, et al. Prognostic Relevance of Integrated Genetic Profiling in Acute Myeloid Leukemia. New England Journal of Medicine. 2012; 366:1079-1089. [PubMed: 22417203]

6. Mrozek K, et al. Prognostic Significance of the European LeukemiaNet Standardized System for Reporting Cytogenetic and Molecular Alterations in Adults With Acute Myeloid Leukemia. J Clin Oncol. 2012 
7. Rai K, et al. Treatment of acute myelocytic leukemia: a study by cancer and leukemia group B. Blood. 1981; 58:1203-1212. [PubMed: 6946847]

8. Fernandez HF, et al. Anthracycline Dose Intensification in Acute Myeloid Leukemia. New England Journal of Medicine. 2009; 361:1249-1259. [PubMed: 19776406]

9. Bisel HF. Criteria for the evaluation of response to treatment in acute leukemia. Blood. 1956; 11:676-677.

10. O’Donnell MR, et al. Acute myeloid leukemia. J Natl Compr Canc Netw. 2012; 10:984-1021. [PubMed: 22878824]

11. Hart JS, Shirakawa S, Trujillo J, Frei E 3rd. The mechanism of induction of complete remission in acute myeloblastic leukemia in man. Cancer Res. 1969; 29:2300-7. [PubMed: 5263333]

12. Cornelissen JJ, et al. The European LeukemiaNet AML Working Party consensus statement on allogeneic HSCT for patients with AML in remission: an integrated-risk adapted approach. Nat Rev Clin Oncol. 2012; 9:579-90. [PubMed: 22949046]

13. Hourigan CS, Karp JE. Development of therapeutic agents for older patients with acute myelogenous leukemia. Curr Opin Investig Drugs. 2010; 11:669-77.

14. Karp JE, et al. Clinical activity of sequential flavopiridol, cytosine arabinoside, and mitoxantrone for adults with newly diagnosed, poor-risk acute myelogenous leukemia. Leuk Res. 2010; 34:87782. [PubMed: 19962759]

15. Karp JE, et al. Randomized phase II study of two schedules of flavopiridol given as timed sequential therapy with cytosine arabinoside and mitoxantrone for adults with newly diagnosed, poor-risk acute myelogenous leukemia. Haematologica. 2012; 97:1736-42. [PubMed: 22733022]

16. Yin JA, et al. Minimal residual disease monitoring by quantitative RT-PCR in core binding factor AML allows risk stratification and predicts relapse: results of the United Kingdom MRC AML-15 trial. Blood. 2012; 120:2826-35. [PubMed: 22875911]

17. Jourdan E, et al. Prospective evaluation of gene mutations and minimal residual disease (MRD) in patients with core binding factor acute myeloid leukemia (CBF-AML). Blood. 2013

18. Todd AV, Ireland CM, Iland HJ. Allele-specific enrichment: a method for the detection of low level N-ras gene mutations in acute myeloid leukemia. Leukemia. 1991; 5:160-1. [PubMed: 2020198]

19. Terstappen LW, Loken MR. Myeloid cell differentiation in normal bone marrow and acute myeloid leukemia assessed by multi-dimensional flow cytometry. Anal Cell Pathol. 1990; 2:22940. [PubMed: 1703434]

20. Kroger N, et al. NCI First International Workshop on the Biology, Prevention, and Treatment of Relapse after Allogeneic Hematopoietic Stem Cell Transplantation: report from the Committee on Disease-Specific Methods and Strategies for Monitoring Relapse following Allogeneic Stem Cell Transplantation. Part I: Methods, acute leukemias, and myelodysplastic syndromes. Biol Blood Marrow Transplant. 2010; 16:1187-211. [PubMed: 20558311]

21. Gulley ML, Shea TC, Fedoriw Y. Genetic tests to evaluate prognosis and predict therapeutic response in acute myeloid leukemia. J Mol Diagn. 2010; 12:3-16. [PubMed: 19959801]

22. Paietta E. Minimal residual disease in acute myeloid leukemia: coming of age. Hematology Am Soc Hematol Educ Program. 2012; 2012:35-42. [PubMed: 23233558]

23. Hokland P, Ommen HB. Towards individualized follow-up in adult acute myeloid leukemia in remission. Blood. 2011; 117:2577-84. [PubMed: 21097673]

24. Hokland P, Ommen HB, Nyvold CG, Roug AS. Sensitivity of minimal residual disease in acute myeloid leukaemia in first remission--methodologies in relation to their clinical situation. $\mathrm{Br} \mathbf{J}$ Haematol. 2012; 158:569-80. [PubMed: 22738609]

25. Bruggemann M, Raff T, Kneba M. Has MRD monitoring superseded other prognostic factors in adult ALL? Blood. 2012

26. Chendamarai E, et al. Role of minimal residual disease monitoring in acute promyelocytic leukemia treated with arsenic trioxide in frontline therapy. Blood. 2012; 119:3413-9. [PubMed: 22374701]

27. Diverio D, et al. Early detection of relapse by prospective reverse transcriptase-polymerase chain reaction analysis of the PML/RARalpha fusion gene in patients with acute promyelocytic leukemia 
enrolled in the GIMEMA-AIEOP multicenter “AIDA” trial. GIMEMA-AIEOP Multicenter

"AIDA" Trial. Blood. 1998; 92:784-9. [PubMed: 9680345]

28. Grimwade D, et al. Prospective minimal residual disease monitoring to predict relapse of acute promyelocytic leukemia and to direct pre-emptive arsenic trioxide therapy. J Clin Oncol. 2009; 27:3650-8. [PubMed: 19506161]

29. Garces-Eisele J. Molecular biology strategies to detect residual disease. Hematology. 2012; 17 (Suppl 1):S66-8. [PubMed: 22507783]

30. Bene MC, Kaeda JS. How and why minimal residual disease studies are necessary in leukemia: a review from WP10 and WP12 of the European LeukaemiaNet. Haematologica. 2009; 94:1135-50. [PubMed: 19586938]

31. Hochhaus A, et al. Quantification of residual disease in chronic myelogenous leukemia patients on interferon-alpha therapy by competitive polymerase chain reaction. Blood. 1996; 87:1549-55. [PubMed: 8608246]

32. Lion $\mathrm{T}$, et al. Use of quantitative polymerase chain reaction to monitor residual disease in chronic myelogenous leukemia during treatment with interferon. Leukemia. 1995; 9:1353-60. [PubMed: 7643624]

33. Druker BJ, et al. Five-year follow-up of patients receiving imatinib for chronic myeloid leukemia. N Engl J Med. 2006; 355:2408-17. [PubMed: 17151364]

34. Hughes TP, et al. Frequency of major molecular responses to imatinib or interferon alfa plus cytarabine in newly diagnosed chronic myeloid leukemia. N Engl J Med. 2003; 349:1423-32. [PubMed: 14534335]

35. Cortes J, Kantarjian H. How I treat newly diagnosed chronic phase CML. Blood. 2012; 120:13907. [PubMed: 22613793]

36. Cross NC, White HE, Muller MC, Saglio G, Hochhaus A. Standardized definitions of molecular response in chronic myeloid leukemia. Leukemia. 2012; 26:2172-5. [PubMed: 22504141]

37. Deininger M. Resistance and relapse with imatinib in CML: causes and consequences. J Natl Compr Canc Netw. 2008; 6 (Suppl 2):S11-S21. [PubMed: 18397677]

38. O'Hare T, Zabriskie MS, Eiring AM, Deininger MW. Pushing the limits of targeted therapy in chronic myeloid leukaemia. Nat Rev Cancer. 2012; 12:513-26. [PubMed: 22825216]

39. Hourigan CS, Levitsky HI. Evaluation of current cancer immunotherapy: hemato-oncology. Cancer J. 2011; 17:309-24. [PubMed: 21952281]

40. Weisser M, et al. Prognostic impact of RT-PCR-based quantification of WT1 gene expression during MRD monitoring of acute myeloid leukemia. Leukemia. 2005; 19:1416-23. [PubMed: 15920493]

41. Tamaki $\mathrm{H}$, et al. Monitoring minimal residual disease in leukemia using real-time quantitative polymerase chain reaction for Wilms tumor gene (WT1). Int J Hematol. 2003; 78:349-56. [PubMed: 14686494]

42. Polak J, et al. Estimation of molecular upper remission limit for monitoring minimal residual disease in peripheral blood of acute myeloid leukemia patients by WT1 expression. Exp Ther Med. 2012; 3:129-133. [PubMed: 22969857]

43. Nowakowska-Kopera A, et al. Wilms' tumor gene 1 expression analysis by real-time quantitative polymerase chain reaction for monitoring of minimal residual disease in acute leukemia. Leuk Lymphoma. 2009; 50:1326-32. [PubMed: 19811333]

44. Cilloni D, et al. Quantitative assessment of WT1 expression by real time quantitative PCR may be a useful tool for monitoring minimal residual disease in acute leukemia patients. Leukemia. 2002; 16:2115-21. [PubMed: 12357365]

45. Cilloni D, et al. Early prediction of treatment outcome in acute myeloid leukemia by measurement of WT1 transcript levels in peripheral blood samples collected after chemotherapy. Haematologica. 2008; 93:921-4. [PubMed: 18443273]

46. Ogawa $\mathrm{H}$, et al. The usefulness of monitoring WT1 gene transcripts for the prediction and management of relapse following allogeneic stem cell transplantation in acute type leukemia. Blood. 2003; 101:1698-704. [PubMed: 12406915] 
47. Ostergaard M, Olesen LH, Hasle H, Kjeldsen E, Hokland P. WT1 gene expression: an excellent tool for monitoring minimal residual disease in $70 \%$ of acute myeloid leukaemia patients - results from a single-centre study. Br J Haematol. 2004; 125:590-600. [PubMed: 15147374]

48. Cilloni $\mathrm{D}$, et al. Real-time quantitative polymerase chain reaction detection of minimal residual disease by standardized WT1 assay to enhance risk stratification in acute myeloid leukemia: a European LeukemiaNet study. J Clin Oncol. 2009; 27:5195-201. [PubMed: 19752335]

49. Ommen HB, et al. Relapse prediction in acute myeloid leukaemia patients in complete remission using WT1 as a molecular marker: development of a mathematical model to predict time from molecular to clinical relapse and define optimal sampling intervals. Br J Haematol. 2008; 141:782-91. [PubMed: 18410450]

50. Polak J, et al. Quantitative monitoring of WT1 expression in peripheral blood before and after allogeneic stem cell transplantation for acute myeloid leukemia - a useful tool for early detection of minimal residual disease. Neoplasma. 2013; 60:74-82. [PubMed: 23067220]

51. Rossi G, et al. Comparison between multiparameter flow cytometry and WT1-RNA quantification in monitoring minimal residual disease in acute myeloid leukemia without specific molecular targets. Leuk Res. 2012; 36:401-6. [PubMed: 22196957]

52. Kwon M, et al. Evaluation of minimal residual disease by real-time quantitative PCR of Wilms' tumor 1 expression in patients with acute myelogenous leukemia after allogeneic stem cell transplantation: correlation with flow cytometry and chimerism. Biol Blood Marrow Transplant. 2012; 18:1235-42. [PubMed: 22281301]

53. Huff V. Wilms' tumours: about tumour suppressor genes, an oncogene and a chameleon gene. Nature Reviews Cancer. 2011; 11:111-121.

54. Miyazaki T, et al. Clinical significance of minimal residual disease detected by multidimensional flow cytometry: serial monitoring after allogeneic stem cell transplantation for acute leukemia. Leuk Res. 2012; 36:998-1003. [PubMed: 22551655]

55. Marcucci G, et al. Prognostic factors and outcome of core binding factor acute myeloid leukemia patients with $\mathrm{t}(8 ; 21)$ differ from those of patients with inv(16): a Cancer and Leukemia Group B study. J Clin Oncol. 2005; 23:5705-17. [PubMed: 16110030]

56. Rubnitz JE, et al. Minimal residual disease-directed therapy for childhood acute myeloid leukaemia: results of the AML02 multicentre trial. Lancet Oncol. 2010; 11:543-52. [PubMed: 20451454]

57. Inaba $\mathrm{H}$, et al. Comparative analysis of different approaches to measure treatment response in acute myeloid leukemia. J Clin Oncol. 2012; 30:3625-32. [PubMed: 22965955]

58. Kronke J, et al. Monitoring of minimal residual disease in NPM1-mutated acute myeloid leukemia: a study from the German-Austrian acute myeloid leukemia study group. J Clin Oncol. 2011; 29:2709-16. [PubMed: 21555683]

59. Kristensen T, Moller MB, Friis L, Bergmann OJ, Preiss B. NPM1 mutation is a stable marker for minimal residual disease monitoring in acute myeloid leukaemia patients with increased sensitivity compared to WT1 expression. Eur J Haematol. 2011; 87:400-8. [PubMed: 21707751]

60. Schnittger S, et al. Minimal residual disease levels assessed by NPM1 mutation-specific RQ-PCR provide important prognostic information in AML. Blood. 2009; 114:2220-31. [PubMed: 19587375]

61. Miglino M, et al. Nucleophosmin gene-based monitoring in de novo cytogenetically normal acute myeloid leukemia with nucleophosmin gene mutations: comparison with cytofluorimetric analysis and study of Wilms tumor gene 1 expression. Leuk Lymphoma. 2012; 53:2214-7. [PubMed: 22475129]

62. Shayegi N, et al. The level of residual disease based on mutant NPM1 is an independent prognostic factor for relapse and survival in AML. Blood. 2013

63. Hou HA, et al. DNMT3A mutations in acute myeloid leukemia: stability during disease evolution and clinical implications. Blood. 2012; 119:559-68. [PubMed: 22077061]

64. Lin LI, et al. Characterization of CEBPA mutations in acute myeloid leukemia: most patients with CEBPA mutations have biallelic mutations and show a distinct immunophenotype of the leukemic cells. Clin Cancer Res. 2005; 11:1372-9. [PubMed: 15746035] 
65. Cloos J, et al. Stability and prognostic influence of FLT3 mutations in paired initial and relapsed AML samples. Leukemia. 2006; 20:1217-20. [PubMed: 16642044]

66. Beierl K, et al. Detection of Minor Clones With Internal Tandem Duplication Mutations of FLT3 Gene in Acute Myeloid Leukemia Using Delta-PCR. Diagn Mol Pathol. 2013; 22:1-9. [PubMed: 23370424]

67. Abdelhamid E, et al. Minimal residual disease monitoring based on FLT3 internal tandem duplication in adult acute myeloid leukemia. Leuk Res. 2012; 36:316-23. [PubMed: 22129478]

68. Grunwald MR, Lin MT, Pratz KW, Gocke CD, Levis MJ. Tandem Duplication PCR (TD-PCR) Is a Novel Method of Detecting Minimal Residual Disease in FLT3/ITD AML and Is Highly Predictive of Relapse Risk Following Allogeneic Transplant. Blood (ASH Annual Meeting Abstracts). 2012:Abstract 2479.

69. Collisson EA, Cho RJ, Gray JW. What are we learning from the cancer genome? Nat Rev Clin Oncol. 2012; 9:621-30. [PubMed: 22965149]

70. Thol F, et al. Next-generation sequencing for minimal residual disease monitoring in acute myeloid leukemia patients with FLT3-ITD or NPM1 mutations. Genes Chromosomes Cancer. 2012; 51:689-95. [PubMed: 22454318]

71. Costa J. Pathology confronts molecular targeted therapies. Nat Clin Pract Oncol. 2006; 3:113. [PubMed: 16520780]

72. Hutchinson L, DeVita VT Jr. The era of personalized medicine: back to basics. Nat Clin Pract Oncol. 2008; 5:623. [PubMed: 18957968]

73. Jordan CT. Targeting the most critical cells: approaching leukemia therapy as a problem in stem cell biology. Nat Clin Pract Oncol. 2005; 2:224-5. [PubMed: 16264940]

74. Gerber JM, et al. A clinically relevant population of leukemic CD34(+)CD38(-) cells in acute myeloid leukemia. Blood. 2012; 119:3571-7. [PubMed: 22262762]

75. Ghiaur G, Gerber J, Jones RJ. Concise review: Cancer stem cells and minimal residual disease. Stem Cells. 2012; 30:89-93. [PubMed: 22045578]

76. Buccisano F, et al. Monitoring of minimal residual disease in acute myeloid leukemia. Curr Opin Oncol. 2009; 21:582-8. [PubMed: 19667983]

77. Nagler A, et al. Detection of minimal residual disease (MRD) after bone marrow transplantation (BMT) by multi-parameter flow cytometry (MPFC). Med Oncol. 1999; 16:177-87. [PubMed: 10523797]

78. Walter RB, et al. Impact of pretransplantation minimal residual disease, as detected by multiparametric flow cytometry, on outcome of myeloablative hematopoietic cell transplantation for acute myeloid leukemia. J Clin Oncol. 2011; 29:1190-7. [PubMed: 21282535]

79. Al-Mawali A, Gillis D, Lewis I. The role of multiparameter flow cytometry for detection of minimal residual disease in acute myeloid leukemia. Am J Clin Pathol. 2009; 131:16-26. [PubMed: 19095561]

80. Feller N, et al. MRD parameters using immunophenotypic detection methods are highly reliable in predicting survival in acute myeloid leukaemia. Leukemia. 2004; 18:1380-90. [PubMed: $15201848]$

81. Freeman SD, Jovanovic JV, Grimwade D. Development of minimal residual disease-directed therapy in acute myeloid leukemia. Semin Oncol. 2008; 35:388-400. [PubMed: 18692689]

82. Kern W, et al. Determination of relapse risk based on assessment of minimal residual disease during complete remission by multiparameter flow cytometry in unselected patients with acute myeloid leukemia. Blood. 2004; 104:3078-85. [PubMed: 15284114]

83. Venditti A, et al. Pretransplant minimal residual disease level predicts clinical outcome in patients with acute myeloid leukemia receiving high-dose chemotherapy and autologous stem cell transplantation. Leukemia. 2003; 17:2178-82. [PubMed: 14576731]

84. Kern W, et al. Prognostic impact of early response to induction therapy as assessed by multiparameter flow cytometry in acute myeloid leukemia. Haematologica. 2004; 89:528-40. [PubMed: 15136215]

85. San Miguel JF, et al. Early immunophenotypical evaluation of minimal residual disease in acute myeloid leukemia identifies different patient risk groups and may contribute to postinduction treatment stratification. Blood. 2001; 98:1746-51. [PubMed: 11535507] 
86. Kern W, Bacher U, Haferlach C, Schnittger S, Haferlach T. The role of multiparameter flow cytometry for disease monitoring in AML. Best Pract Res Clin Haematol. 2010; 23:379-90. [PubMed: 21112037]

87. Zeleznikova T, Stevulova L, Kovarikova A, Babusikova O. Increased myeloid precursors in regenerating bone marrow; implications for detection of minimal residual disease in acute myeloid leukemia. Neoplasma. 2007; 54:471-7. [PubMed: 17949229]

88. Tomova A, Babusikova O. Shifts in expression of immunological cell markers in relapsed acute leukemia. Neoplasma. 2001; 48:164-8. [PubMed: 11583283]

89. Baer MR, et al. High frequency of immunophenotype changes in acute myeloid leukemia at relapse: implications for residual disease detection (Cancer and Leukemia Group B Study 8361). Blood. 2001; 97:3574-80. [PubMed: 11369653]

90. Macedo A, et al. Phenotypic changes in acute myeloid leukaemia: implications in the detection of minimal residual disease. J Clin Pathol. 1996; 49:15-8. [PubMed: 8666678]

91. Langebrake $\mathrm{C}$, et al. Immunophenotypic differences between diagnosis and relapse in childhood AML: Implications for MRD monitoring. Cytometry B Clin Cytom. 2005; 63:1-9. [PubMed: 15624201]

92. Al-Mawali A, Gillis D, Hissaria P, Lewis I. Incidence, sensitivity, and specificity of leukemiaassociated phenotypes in acute myeloid leukemia using specific five-color multiparameter flow cytometry. Am J Clin Pathol. 2008; 129:934-45. [PubMed: 18480011]

93. Loken MR, et al. Residual disease detected by multidimensional flow cytometry signifies high relapse risk in patients with de novo acute myeloid leukemia: a report from Children's Oncology Group. Blood. 2012; 120:1581-1588. [PubMed: 22649108]

94. Wood BL. Myeloid malignancies: myelodysplastic syndromes, myeloproliferative disorders, and acute myeloid leukemia. Clin Lab Med. 2007; 27:551-75. vii. [PubMed: 17658407]

95. Buccisano F, et al. Cytogenetic and molecular diagnostic characterization combined to postconsolidation minimal residual disease assessment by flow cytometry improves risk stratification in adult acute myeloid leukemia. Blood. 2010; 116:2295-303. [PubMed: 20548095]

96. van Rhenen A, et al. Aberrant marker expression patterns on the CD34+CD38- stem cell compartment in acute myeloid leukemia allows to distinguish the malignant from the normal stem cell compartment both at diagnosis and in remission. Leukemia. 2007; 21:1700-7. [PubMed: 17525725]

97. Anguille S, Van Tendeloo VF, Berneman ZN. Leukemia-associated antigens and their relevance to the immunotherapy of acute myeloid leukemia. Leukemia. 2012; 26:2186-96. [PubMed: 22652755]

98. Biernacki MA, et al. Efficacious immune therapy in chronic myelogenous leukemia (CML) recognizes antigens that are expressed on CML progenitor cells. Cancer Res. 2010; 70:906-15. [PubMed: 20103624]

99. Carter BZ, et al. Survivin is highly expressed in CD34(+)38(-) leukemic stem/progenitor cells and predicts poor clinical outcomes in AML. Blood. 2012; 120:173-80. [PubMed: 22645176]

100. Du W, Li XE, Sipple J, Pang Q. Overexpression of IL-3Ralpha on CD34+CD38- stem cells defines leukemia-initiating cells in Fanconi anemia AML. Blood. 2011; 117:4243-52. [PubMed: 21330473]

101. Gerber JM, et al. Characterization of chronic myeloid leukemia stem cells. Am J Hematol. 2011; 86:31-7. [PubMed: 21132730]

102. Jan M, et al. Clonal evolution of preleukemic hematopoietic stem cells precedes human acute myeloid leukemia. Sci Transl Med. 2012; 4:149ra118.

103. Oehler VG, et al. The preferentially expressed antigen in melanoma (PRAME) inhibits myeloid differentiation in normal hematopoietic and leukemic progenitor cells. Blood. 2009; 114:3299308. [PubMed: 19625708]

104. Dominietto A. Minimal residual disease markers before and after allogeneic hematopoietic stem cell transplantation in acute myeloid leukemia. Curr Opin Hematol. 2011; 18:381-7. [PubMed: 21986564]

105. Pozzi S, et al. Leukaemia relapse after allogeneic transplants for acute myeloid leukaemia: predictive role of WT1 expression. Br J Haematol. 2013; 160:503-9. [PubMed: 23294252] 
106. Stahl T, et al. Minimal residual disease diagnostics in patients with acute myeloid leukemia in the post-transplant period: comparison of peripheral blood and bone marrow analysis. Leuk Lymphoma. 2010; 51:1837-43. [PubMed: 20849383]

107. Stone RM. Should the presence of minimal residual disease (MRD) in morphologic complete remission alter post-remission strategy in AML? Best Pract Res Clin Haematol. 2011; 24:50914. [PubMed: 22127313]

108. Wayne AS, Radich JP. Pretransplant MRD: the light is yellow, not red. Blood. 2012; 120:244-6. [PubMed: 22791775]

109. Fang M, et al. Prognostic impact of discordant results from cytogenetics and flow cytometry in patients with acute myeloid leukemia undergoing hematopoietic cell transplantation. Cancer. 2012; 118:2411-9. [PubMed: 21928360]

110. Zhao XS, et al. Wilms' tumor gene 1 expression: an independent acute leukemia prognostic indicator following allogeneic hematopoietic SCT. Bone Marrow Transplant. 2012; 47:499-507. [PubMed: 21643023]

111. Scheffold C, et al. Prediction of relapse of acute myeloid leukemia in allogeneic transplant recipients by marrow CD34+ donor cell chimerism analysis. Leukemia. 2004; 18:2048-50. [PubMed: 15470491]

112. Bornhauser M, et al. Monitoring of donor chimerism in sorted CD34+ peripheral blood cells allows the sensitive detection of imminent relapse after allogeneic stem cell transplantation. Haematologica. 2009; 94:1613-7. [PubMed: 19880783]

113. Platzbecker U, et al. Azacitidine for treatment of imminent relapse in MDS or AML patients after allogeneic HSCT: results of the RELAZA trial. Leukemia. 2012; 26:381-9. [PubMed: 21886171]

114. Sockel K, et al. Minimal residual disease-directed preemptive treatment with azacitidine in patients with NPM1-mutant acute myeloid leukemia and molecular relapse. Haematologica. 2011; 96:1568-70. [PubMed: 21750085]

115. Yan $\mathrm{CH}$, et al. Risk stratification-directed donor lymphocyte infusion could reduce relapse of standard-risk acute leukemia patients after allogeneic hematopoietic stem cell transplantation. Blood. 2012; 119:3256-62. [PubMed: 22337715]

116. Zhu H-H, et al. MRD-directed risk-stratification treatment may improve outcome of $\mathrm{t}(8 ; 21)$ AML in the first complete remission: results from AML05 Multicenter Trial. Blood. 2013

117. Ossenkoppele G, Schuurhuis GJ. MRD in AML: time for redefinition of CR? Blood. 2013; 121:2166-8. [PubMed: 23520326]

118. Ommen HB, et al. Strikingly different molecular relapse kinetics in NPM1c, PML-RARA, RUNX1-RUNX1T1, and CBFB-MYH11 acute myeloid leukemias. Blood. 2010; 115:198-205. [PubMed: 19901261]

119. Ommen HB, Roug AS, Hokland P. The minimal residual disease concept coming of age - now for the direct comparison of methodologies. Leuk Res. 2012; 36:392-3. [PubMed: 22261230]

120. Rajkumar SV. Haematological cancer: Lenalidomide maintenance[mdash]perils of a premature denouement. Nat Rev Clin Oncol. 2012; 9:372-374. [PubMed: 22665364]

121. Salles G, et al. Rituximab maintenance for 2 years in patients with high tumour burden follicular lymphoma responding to rituximab plus chemotherapy (PRIMA): a phase 3, randomised controlled trial. Lancet. 2011; 377:42-51. [PubMed: 21176949]

122. Kirk R. Lung cancer: Maintenance chemotherapy--a stitch in time saves nine? Nat Rev Clin Oncol. 2012; 9:187. [PubMed: 22392096]

123. Barrett J, Rezvani K. Immunotherapy: Can we include vaccines with stem-cell transplantation? Nat Rev Clin Oncol. 2009; 6:503-5. [PubMed: 19707241]

124. Quintas-Cardama A, et al. Epigenetic therapy is associated with similar survival compared with intensive chemotherapy in older patients with newly diagnosed acute myeloid leukemia. Blood. 2012

125. Zhu YX, Kortuem KM, Stewart AK. Molecular mechanism of action of immune-modulatory drugs thalidomide, lenalidomide and pomalidomide in multiple myeloma. Leuk Lymphoma. 2012

126. Kalos M, et al. T cells with chimeric antigen receptors have potent antitumor effects and can establish memory in patients with advanced leukemia. Sci Transl Med. 2011; 3:95ra73. 
127. Barrett AJ, Battiwalla M. Relapse after allogeneic stem cell transplantation. Expert Rev Hematol. 2010; 3:429-41. [PubMed: 21083034]

128. Disis ML, Bernhard H, Jaffee EM. Use of tumour-responsive T cells as cancer treatment. Lancet. 2009; 373:673-83. [PubMed: 19231634]

129. Grimwade D. The changing paradigm of prognostic factors in acute myeloid leukaemia. Best Pract Res Clin Haematol. 2012; 25:419-25. [PubMed: 23200538]

130. Coustan-Smith E, Campana D. Should evaluation for minimal residual disease be routine in acute myeloid leukemia? Curr Opin Hematol. 2013; 20:86-92. [PubMed: 23380603]

131. Buccisano F, et al. Prognostic and therapeutic implications of minimal residual disease detection in acute myeloid leukemia. Blood. 2012; 119:332-41. [PubMed: 22039260]

132. Carlson KS, Guzman ML. Is Minimal Residual Disease Monitoring Clinically Relevant in Adults with Acute Myelogenous Leukemia? Curr Hematol Malig Rep. 2013

133. DiNardo CD, Luger SM. Beyond morphology: minimal residual disease detection in acute myeloid leukemia. Curr Opin Hematol. 2012; 19:82-8. [PubMed: 22314322]

134. Grimwade D, Vyas P, Freeman S. Assessment of minimal residual disease in acute myeloid leukemia. Curr Opin Oncol. 2010; 22:656-63. [PubMed: 20805746]

135. Hourigan CS, Karp JE. New considerations in the design of clinical trials for the treatment of acute leukemia. Clin Investig (Lond). 2011; 1:509-517.

136. Cheson BD, et al. Revised recommendations of the International Working Group for Diagnosis, Standardization of Response Criteria, Treatment Outcomes, and Reporting Standards for Therapeutic Trials in Acute Myeloid Leukemia. J Clin Oncol. 2003; 21:4642-9. [PubMed: 14673054]

137. Chen Y, et al. Persistence of cytogenetic abnormalities at complete remission after induction in patients with acute myeloid leukemia: prognostic significance and the potential role of allogeneic stem-cell transplantation. J Clin Oncol. 2011; 29:2507-13. [PubMed: 21555694]

\section{Biographies}

Christopher S. Hourigan is Principal Investigator of the Myeloid Malignancies Section, Hematology Branch at the National Heart, Lung and Blood Institute of the National Institutes of Health. He received both his medical degree and D. Phil. in human immunology from Oxford University and then completed residency training in medicine at Guy's and St. Thomas' Hospital in London and the Johns Hopkins Bayview Medical Center in Baltimore, clinical fellowships in Medical Oncology at Johns Hopkins Hospital and in Hematology at NHLBI, and a research fellowship from the Immunology and Immunotherapy research program of the Sidney Kimmel Comprehensive Cancer Center at Johns Hopkins. His research interest is the human immune response to acute myeloid leukemia.

Judith E. Karp is Director of the Leukemia Program at the Johns Hopkins Sidney Kimmel Comprehensive Cancer Center and Professor of Oncology and Professor of Medicine at the Johns Hopkins School of Medicine. She received her medical degree from Stanford University School of Medicine, completed internship and residency at Stanford University Hospital and Johns Hopkins Hospital, and completed her Oncology Fellowship at the Johns Hopkins University School of Medicine. From 1990-1995, she served as Special Assistant to Director, National Cancer Institute. Her research interests focused on the experimental therapeutics of acute leukemias, including development of timed sequential therapy, new biologic agents for older adults with acute leukemias, and new approaches to the treatment of refractory acute leukemias including secondary leukemias that evolve from myelodysplasia or from prior cytotoxic chemotherapies. 


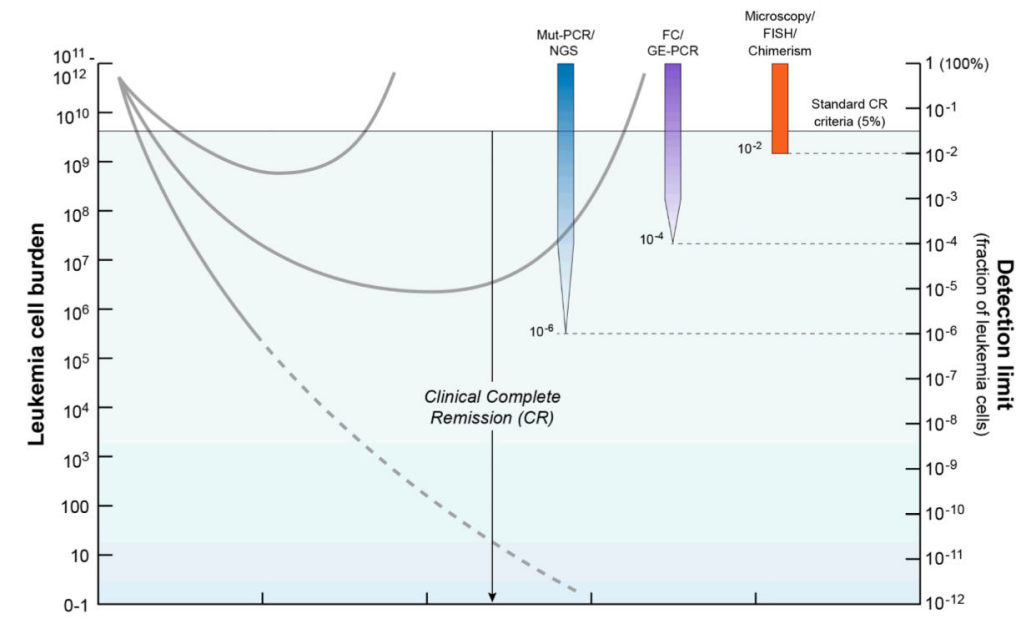

Figure 1. Detection thresholds of various MRD modalities compared to traditional clinical complete remission

Axis scales approximate and solely for illustrative purposes.

http://www.nature.com/nrclinonc/journal/v10/n8/fig_tab/nrclinonc.2013.100_F1.html and http://www.nature.com/nrclinonc/journal/v10/n8/fig_tab/nrclinonc.2013.100_F2.html 


\section{Table 1}

Evolution of response criteria for complete remission in adult patients with Acute Myeloid Leukemia.

\begin{tabular}{|l|c|c|}
\hline & \multicolumn{1}{|c|}{$\mathbf{1 9 5 6}^{\mathbf{9}}$} & $\mathbf{2 0 1 2}^{\mathbf{1 0}}$ \\
\hline Bone Marrow & $\begin{array}{c}\text { Less than 5\% blasts and absence of cells that can be individually } \\
\text { identified as leukemic. }\end{array}$ & $\begin{array}{c}\text { Less than 5\% blasts including no blasts with } \\
\text { Auer rods. }\end{array}$ \\
\hline Extramedullary disease & Subsidence of all evidence of leukemic infiltration & No residual evidence of extramedullary disease \\
\hline Platelets & $>100,000$ & $>100,000$ \\
\hline Neutrophil count & $>200 / \mathrm{mm}^{3}$ & $>1000 / \mathrm{mcL}$ \\
\hline Hemoglobin & $>12 \mathrm{gm} / \mathrm{dl}$ for 1 month & Transfusion-free \\
\hline Clinical & No symptoms ascribable to leukemia & No criteria. \\
\hline
\end{tabular}

An additional category of "cytogenetics normal in those with previously abnormal cytogenetics" is listed but not required for a morphological complete response in the complete remission category of $2012 \mathrm{NCCN}$ guidelines 10 and is "commended primarily for use in clinical research studies" in the current international working group (IWG) criteria 136 that those guidelines are based on. There is evidence however that persistence of cytogenetic abnormalities in AML patients in complete remission is associated with a worse prognosis 137 . 
Table 2

Comparison of Flow Cytometry versus PCR for detection of MRD in AML

\begin{tabular}{|c|c|}
\hline Flow Cytometry & Polymerase Chain Reaction \\
\hline $\begin{array}{l}\text { Requires significant human capital as highly trained pathologist } \\
\text { expertise required for interpretation. }\end{array}$ & $\begin{array}{l}\text { Can be run in any certified laboratory with real-time PCR capacity } \\
\text { including off-site central reference laboratory. }\end{array}$ \\
\hline Results within one day & Results may take several days \\
\hline Single result interpretable & Interpretation often requires trend of results \\
\hline Excellent specificity when using defined LAIP & $\begin{array}{c}\text { Low level transcripts in remission samples common - requires setting of } \\
\text { threshold limits. }\end{array}$ \\
\hline $\begin{array}{l}\text { Requires use multiple LAIPs, multiple antibody flurochrome } \\
\text { combinations and at least 200,000 events for adequate sensitivity. }\end{array}$ & $\begin{array}{c}\text { Excellent sensitivity, at least equal and in some cases superior to flow } \\
\text { cytometry. }\end{array}$ \\
\hline $\begin{array}{l}\text { Leukemic phenotype may "change" over time, initial LAIP may } \\
\text { not identify subclones responsible for relapse. }\end{array}$ & Many targets stable \\
\hline Allows detection of cells with leukemia stem cell phenotype. & Does not allow discrimination of "bulk" from "stem cell" leukemia. \\
\hline Detection to $10^{-4}$ possible & Detection from $10^{-4}$ to $10^{-6}$ possible \\
\hline
\end{tabular}




\begin{tabular}{|c|c|c|c|c|c|c|c|c|c|c|c|}
\hline$\approx$ & $\therefore i n$ & 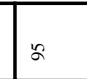 & $\infty$ & 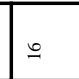 & $\therefore$ & $\bar{n}$ & $\cong$ & $\cong$ & 2 & $=$ & $\cong$ \\
\hline $\mid$ & 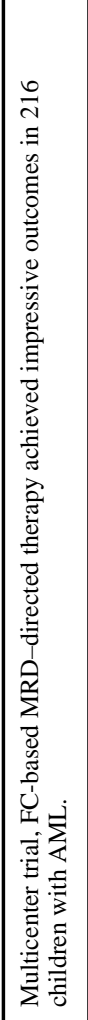 & 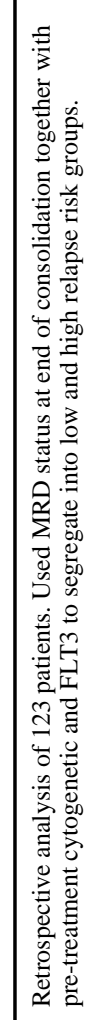 & 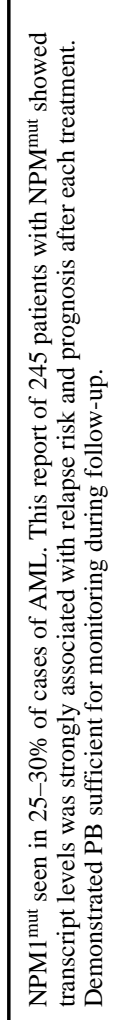 & 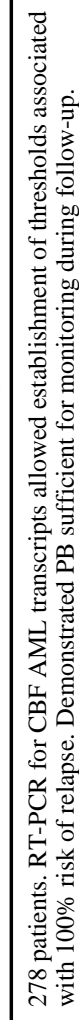 & 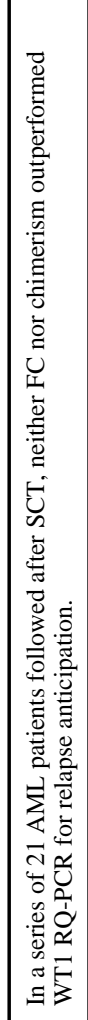 & 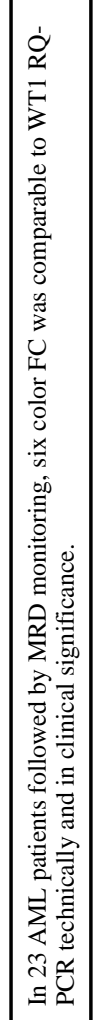 & 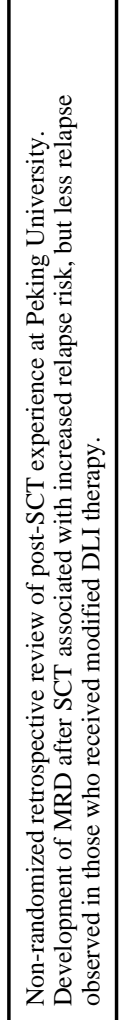 & 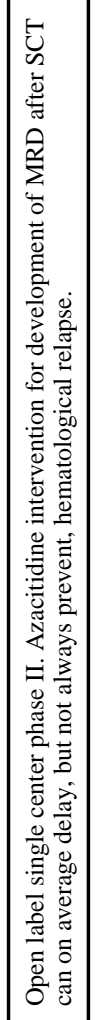 & 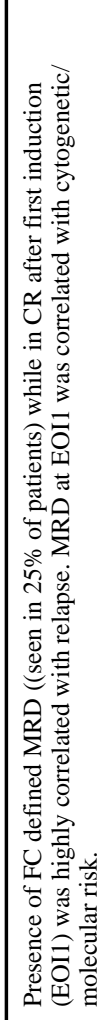 & 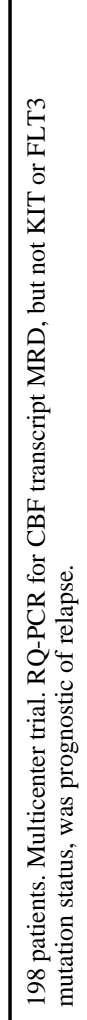 & 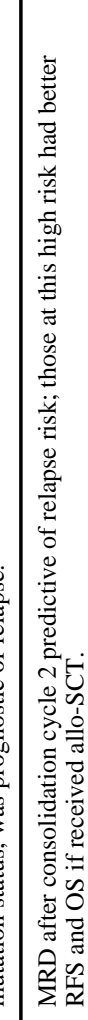 \\
\hline 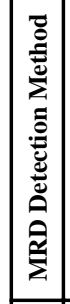 & 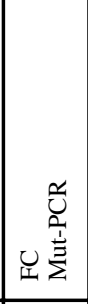 & 足 & & 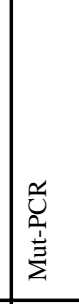 & 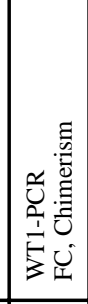 & \begin{tabular}{|l|} 
\\
0 \\
0 \\
0 \\
5 \\
3 \\
\end{tabular} & 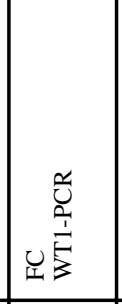 & 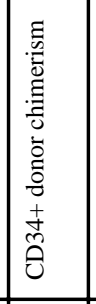 & 足 & 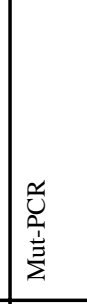 & 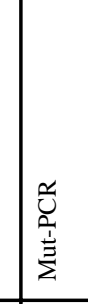 \\
\hline 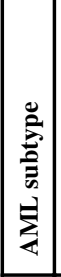 & 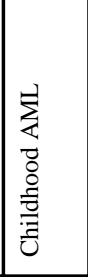 & 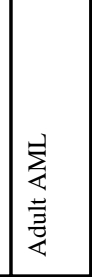 & 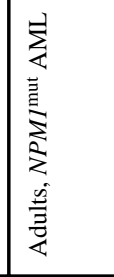 & 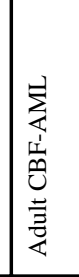 & 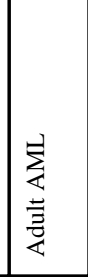 & 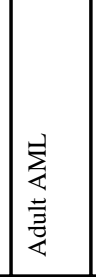 & 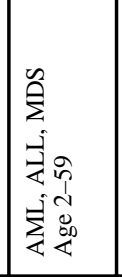 & 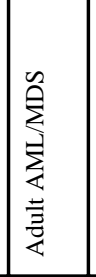 & 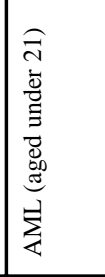 & 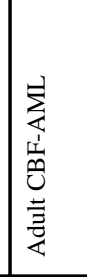 & 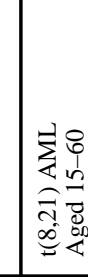 \\
\hline 竎 & वेंते & 过 & 高 & ते & 察 & 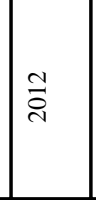 & $\overline{\tilde{c}}$ & 寄 & 高 & $\vec{\vdots}$ & $\stackrel{m}{\stackrel{N}{N}}$ \\
\hline 氶 & 妾 & 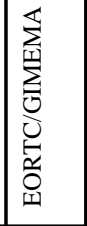 & 产 & 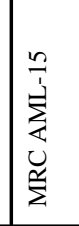 & 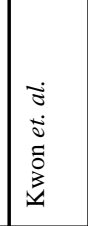 & 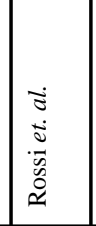 & 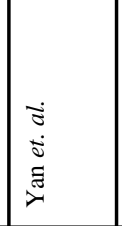 & 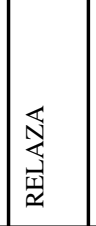 & 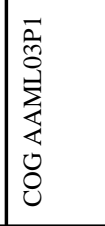 & 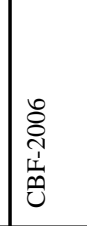 & 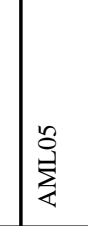 \\
\hline
\end{tabular}




\section{Table 4}

Clinical scenarios in the care of AML patients where MRD may have utility.

- High-resolution determination of efficacy of therapy

- To allow target-driven titration of dose and duration of treatment

- Relapse risk stratification after induction to allow triage to optimal consolidation therapy

- To determine prognosis after completion of standard treatment

- To spare toxicity and cost of SCT in those with low risk of relapse

- Assignment to maintenance therapy after completion of standard treatment 